\title{
Evaluating the mainstream impact of ophthalmological research with Google Trends
}

\author{
Arun James Thirunavukarasu (i] ${ }^{1,2}$
}

Received: 28 August 2020 / Revised: 22 September 2020 / Accepted: 22 October 2020 / Published online: 1 November 2020

(c) The Royal College of Ophthalmologists 2020

\section{To the Editor:}

Google Trends is a cost-effective means of assaying relative public interest-based on the frequency of Google search requests-in a topic for a defined geographical region and time period [1]. Its investigational utility depends on comprehensive reporting: queries' search terms, comparisons, and geographical and time ranges must be stated to maximise reproducibility and the accuracy of interpretation [2]. Here, Google interest was compared to PubMed publication frequency between 2004 and 2020, to explore whether or not ophthalmological research has an impact on interest in the general public. It was hypothesised that any impact would manifest as correlation between the two variables.

Using Google Trends, 200 monthly readings of worldwide Google interest between January 2004-August 2020, inclusive, were obtained for eight common ophthalmological conditions: "amblyopia", "cataract", "diabetic retinopathy", "glaucoma", "hyperopia", "macular degeneration", "myopia" and "strabismus". Glaucoma was selected as a common reference across queries, as it had the greatest interest of the queried terms. On PubMed, the number of publications related to the same eight terms was obtained for each of the 17 years between 2004 and 2020, inclusive. Queries were made on August 24th, 2020. Google interest and PubMed publications were plotted against time, and against each other, to visualise the extent of association between the two variables. As statistically significant $(p<$ 0.05 ) deviations from normality were recorded for all search terms in Shapiro-Wilks tests, correlation was quantified by calculation of Kendall's rank correlation coefficient, $\tau$. Data visualisation and statistical analysis were conducted in $R$ (v3.6.1) and Affinity Designer (v1.8.4).

Arun James Thirunavukarasu

ajt205@cam.ac.uk

1 Corpus Christi College, University of Cambridge, Cambridge, UK
Google interest in ophthalmological conditions was greatest between 2004 and 2005, falling to a minimum around 2011 with a subsequent rise to the present day interrupted by a sharp drop in March 2020 (Fig. 1), likely due to disproportionate interest in the Covid-19 pandemic. In contrast, PubMed publications tended to grow steadily, with a drop in 2020 (Fig. 1), attributable to both the pandemic and lack of four months' research relative to other years. A scatter plot relating the two variables (Fig. 2) indicated weak positive correlation overall, with most points lying outside the $95 \%$ confidence intervals of the best-fit trend-line. No correlation was apparent within search terms. Correlation coefficients agreed with these deductions, with overall positive correlation $(\tau=0.620$, $p=1.35 \mathrm{E}-26)$ and no significant correlations $(p>0.05)$ within search terms.

The overall correlation between Google interest and PubMed publications indicates concordance between the interests of the scientific community and general public. However, lack of correlation within conditions suggests that ophthalmological research has little direct effect on laypeople's interests, which may instead be closer related to the prevalence of the respective conditions. Correlation could also be masked by the lack of normalisation of PubMed data: ophthalmology may not have kept up with inflation observed across the scientific literature over time. Further investigations could explore the effect on Google interest of high impact publications, or publication-inspired news pieces. Similar ophthalmological event analyses have previously been conducted, evaluating the effect of public health campaigns [3], conjunctivitis epidemics [4] and Bono developing glaucoma [5].

2 School of Clinical Medicine, University of Cambridge, Cambridge, UK 

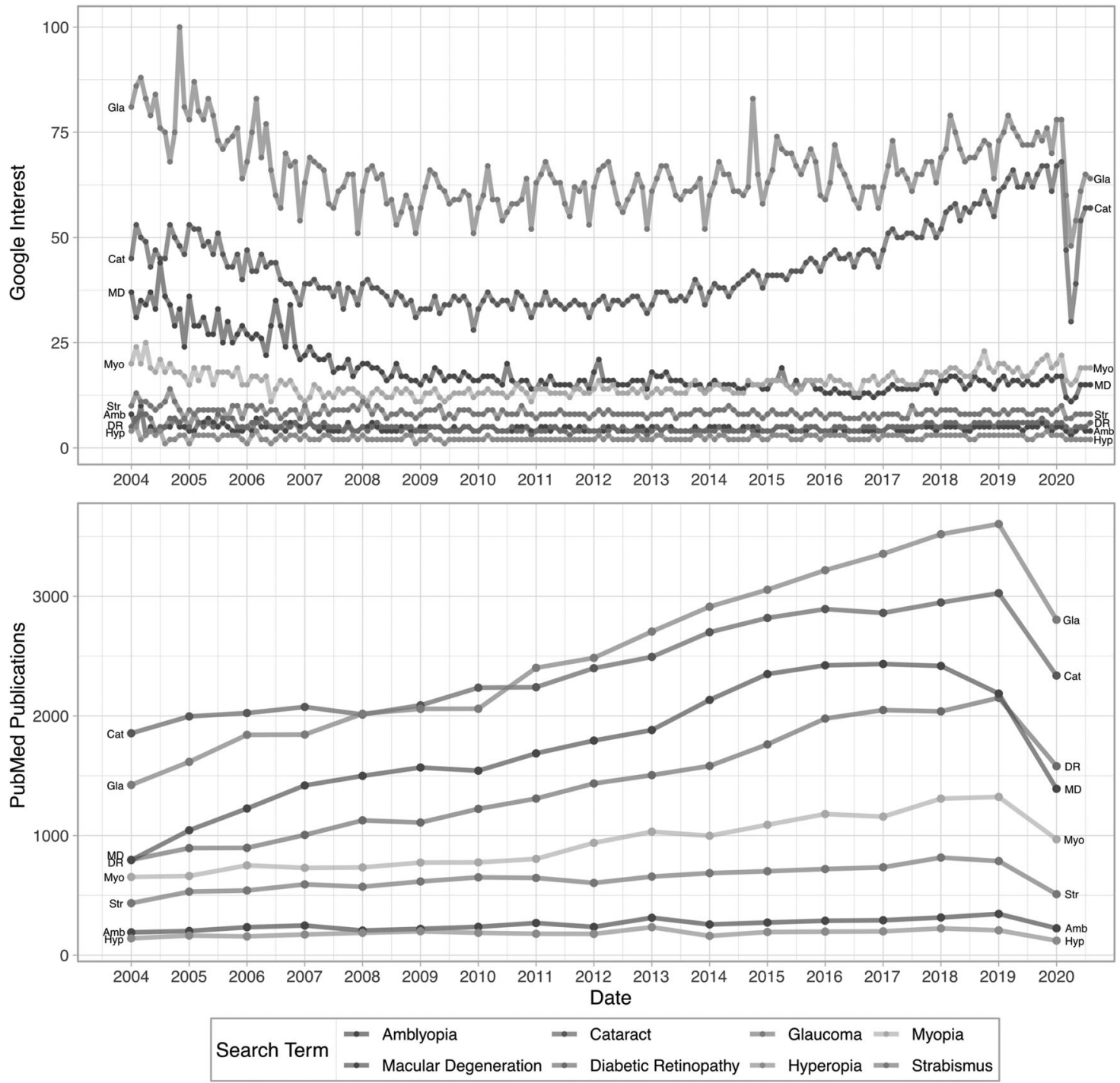

Fig. 1 Google interest (top) and PubMed publications (bottom) plotted against time between January 2004 and August 2020. Amb amblyopia, Cat cataract, DR diabetic retinopathy, Gla glaucoma, Hyp hyperopia, MD macular degeneration, Myo myopia, Sra strabismus.

Fig. 2 Scatter plot relating Google interest to PubMed publications. Convex hulls define the regions within which points relating to each ophthalmological term are grouped. Amb amblyopia, Cat cataract, DR diabetic retinopathy, Gla glaucoma, Hyp hyperopia, MD macular degeneration, Myo myopia, Sra strabismus.

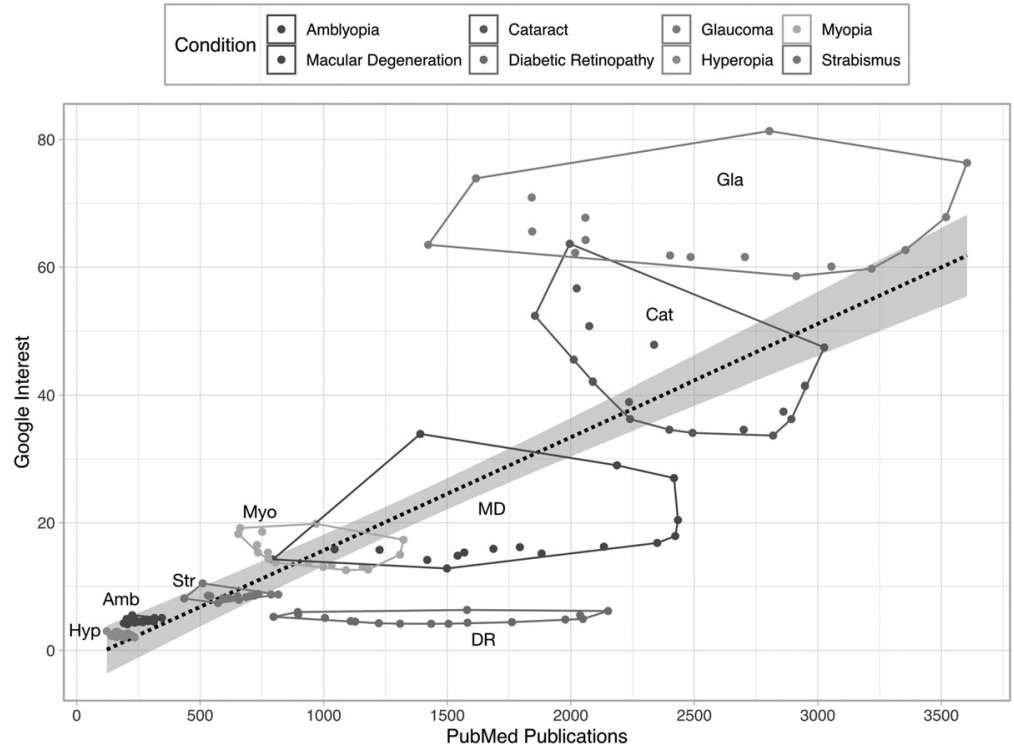




\section{Compliance with ethical standards}

Conflict of interest The author declares no conflict of interest.

Publisher's note Springer Nature remains neutral with regard to jurisdictional claims in published maps and institutional affiliations.

\section{References}

1. Rasheed R, Sivaprasad S. Google trends as a surrogate marker of public awareness of diabetic retinopathy. Eye. 2020;34:1010-2.
2. Nuti SV, Wayda B, Ranasinghe I, Wang S, Dreyer RP, Chen SI, et al. The use of google trends in health care research: a systematic review. PLOS ONE. 2014;9:e109583.

3. Kho A, Niederer R, Wilson G. Exploring the impact of public health campaigns for glaucoma and macular degeneration utilising Google Trends data in a New Zealand setting. N. Z. Med J. 2020; 133:10-5.

4. Deiner MS, McLeod SD, Wong J, Chodosh J, Lietman TM, Porco TC. Google searches and detection of conjunctivitis epidemics worldwide. Ophthalmology 2019;126:1219-29.

5. Lyons C, Ellard R, McElnea E, Townley DI. Still haven't found what i'm looking for... Bono, google and glaucoma awareness. Ir Med J. 2017;110:568. 